\title{
Transpiração em espécie de grande porte na Floresta Nacional de Caxiuanã, Pará ${ }^{1}$
}

\author{
Rafael F. da Costa ${ }^{2}$, Vicente de P. R. da Silva ${ }^{2}$, Maria L. P. Ruivo ${ }^{3}$, Patrick Meir ${ }^{4}$, Antonio C. L. Costa ${ }^{5}$, Yadvinder S. Malhi' \\ Alan P. Braga ${ }^{5}$, Paulo H. L. Gonçalves ${ }^{5}$, João de A. Silva Jr. ${ }^{5}$ \& John Grace $^{4}$
}

\begin{abstract}
RESUMO
Durante o experimento "O Impacto da Seca Prolongada nos Fluxos de Água e Dióxido de Carbono em uma Floresta Tropical Amazônica" (ESECAFLOR) realizou-se este trabalho. Trata-se de um subprojeto do Experimento de Grande escala da BiosferaAtmosfera da Amazônia (LBA), localizado na Estação Científica Ferreira Pena, dentro da Floresta Nacional de Caxiuanã, Pará ( $1^{\circ} 42^{\prime} 30^{\prime \prime} \mathrm{S} ; 51^{\circ} 31^{\prime} 45^{\prime \prime} \mathrm{W} ; 62 \mathrm{~m}$ altitude). A região tem floresta bem preservada, com dossel médio de $35 \mathrm{~m}$. As espécies predominantes em terra-firme, são: Eschweilera coriacea (Mata-matá branco), Voucapoua americana (Acapu) e Protium pallidum (Breu Branco). Medidas foram realizadas entre 03 a 16 de dezembro de 2000 e 12 a 25 de janeiro de 2003, objetivandose determinar a transpiração de dois exemplares de Eschweilera coriacea, mediante os efeitos da seca provocada. A área do ESECAFLOR compreende duas parcelas, cada uma com 1 ha, parcela A (controle) e parcela B (exclusão da chuva). Para o fluxo de seiva, o método foi o Balanço de Calor no Tronco, com sistema Sap Flow meter, P4.1; entre os períodos analisados, a transpiração média registrou aumento de 56\% na árvore A237 (parcela A) e redução de 68\% na árvore B381 (parcela B).
\end{abstract}

Palavras-chave: amazônia, Eschweilera coriacea, fluxo de seiva, LBA

\section{Transpiration in large size species in Caxiuanã National Forest, in the State of Pará, Brazil}

\begin{abstract}
During the "Long-term of impact drought on water and carbon dioxide fluxes in Amazonian Tropical Rainforest Experiment" (ESECAFLOR), this study was carried out, which is a subproject of Large Scale Biosphere Atmosphere Experiment in Amazônia (LBA), located in the Ferreira Penna Scientific Station (FPSS) in the Caxiuanã National Forest (CNF) in Pará State ( $1^{\circ} 42^{\prime} 30^{\prime \prime}$ $S$; $51^{\circ} 31^{\prime} 45^{\prime \prime} \mathrm{W} ; 62 \mathrm{~m}$ altitude). The region has a well-preserved forest, with canopy of $35 \mathrm{~m}$. The predominate tree species in the landscape are Eschweilera coriacea (White Matá-matá), Voucapoua americana (Acapu) and Protium pallidum (White Pitch). Sap flow measurements were made in the wet season (03-16 December 2000 and 12-25 January 2003), to evidence the effect of long term induced drought, aiming to determinate the transpiration of Eschweilera coriacea. The ESECAFLOR site consists of two different areas with 1 ha each. Plot A (control) and Plot B (rainfall exclusion). The Trunk Heat Balance (THB) method was applied to sap flow measurements, by Sap Flow Meter P4.1 system. Between analysed periods, the mean transpiration of E. Coriacea increased 56\% in the tree A237 (control plot) and decreased 68\% in B381 (drought plot).
\end{abstract}

Key words: Amazon, Eschweilera coriacea, LBA, sap flow

1 Parte da Tese de Doutorado em Meteorologia do primeiro autor pela UFCG

${ }^{2}$ UACA/UFCG, Av. Aprígio Veloso 822, Bodocongó, CEP 58.109-970, Campina Grande, PB, Fone: (83) 3333 1054. E-mail: rfcostampeg@bol.com.br; vicente@dca.ufcr.edu.br

3 CCTE/MPEG, Coordenação de Ciências da Terra e Ecologia, Museu Paraense Emilio Goeldi, Av. Perimetral 1901, Terra-Firme, CP. 399, CEP 66077-530,

Belém, PA. Fone: (91) 3217 6153. E-mail: ruivo@museu-goeldi.br

${ }^{4}$ University of Edinburgh, School of Geosciences, Geography Building, Drummond Street, Edinburgh EH8 9XP, Scotland, UK, Fone: (44) 1316502521.

E-mail: pmeir@geo.ed.ac.uk; J.Grace@ed.ac.uk

5 DM/UFPA, Av. Augusto Corrêa 1, Guamá, CEP 66075-110, Belém, PA. Fone: (91) 32017207. E-mail: lola@ufpa.br; alan_meteoro@yahoo.com.br; paulofuracao@zipmail.com.br; meteoro.joao@zipmail.com.br

${ }^{6}$ University of Oxford, School of Geography and the Environment, Oxford OX1 3QY, England, UK. Fone: (44) 18652719 13. E-mail: ymalhi@ed.ac.uk 


\section{INTRODUÇÃO}

O Experimento 'O Impacto da Seca Prolongada nos Fluxos de Água e Dióxido de Carbono em uma Floresta Tropical Amazônica' (ESECAFLOR) está sendo desenvolvido como parte integrante do 'Experimento de Grande Escala da Biosfera-Atmosfera na Amazônia' (LBA), na Floresta Nacional (FLONA) de Caxiuanã, no Estado do Pará.

O LBA tem, como objetivo principal, o entendimento das alterações nos ciclos da água, carbono e nutrientes, e os balanços de energia solar, em função das modificações verificadas na cobertura vegetal da região. Este projeto trata dos relacionamentos entre o clima, o solo, os gases atmosféricos e os impactos do uso da terra na Amazônia; além disso ainda estuda os processos globais dos ciclos biogeoquímicos existentes entre a biosfera e a atmosfera.

O ESECAFLOR é uma proposta de avaliação das conseqüências provocadas no ecossistema por um longo período de exclusão de parte significante da água da chuva, que seria como a simulação de um evento de El Niño, que provoca seca anômala em determinadas áreas na Amazônia. A ocorrência de estresse hídrico, em florestas tropicais, causa alterações ainda pouco conhecidas em todo o ecossistema (Costa et al., 2003a).

Diversos estudos são desenvolvidos com o objetivo de determinar medidas e/ou estimativas de evapotranspiração. Grande parte deste esforço foi direcionada para as espécies frutíferas de pomar ou para culturas com produção em grande escala, através da aplicação de diferentes métodos (Casa et al., 2000; Netto et al., 2000; Silva et al., 2000; Azevedo et al., 2003; Möller et al., 2004; Sammis et al., 2004; Testi et al., 2004; Williams et al., 2004; Nicolasa et al., 2005; Orgaz et al., 2005); entretanto, a determinação da evapotranspiração em espécies florestais tem sido menos enfocada porém não menos importante (Meinzer et al., 1997, Naves-Barbiero et al., 2000, Köstner, 2001). Foram realizados, também, alguns estudos para estimativa de evapotranspiração na região amazônica (Villa Nova et al., 1976; Marques et al., 1980; Shuttleworth et al., 1987).

Em todo o mundo, as florestas, sobretudo as tropicais, passam por processos de grande ocupação e extrativismo exacerbado mas, pouco se sabe sobre qual será o limite suportável por essas áreas em relação às ações antrópicas. Mudanças climáticas provocadas por secas prolongadas e aquecimento global também interferem nos fluxos de vapor d'água em florestas no planeta. Malhi et al. (2002) sugeriram alguns procedimentos que, se postos em prática, minimizarão os impactos antropogênicos nas florestas tropicais e permitirão melhor qualidade ambiental, principalmente no que se refere à redução das emissões de dióxido de carbono $\left(\mathrm{CO}_{2}\right)$ para a atmosfera.

A capacidade de absorção de água pelas raízes das plantas, sob diferentes condições de solo, salinidade e nutrição, está associada ao poder dessas raízes de atingir elevadas taxas de respiração. A atividade estomática responde às mudanças das condições climáticas independentes da região. A breve redução na radiação solar incidente sobre uma árvore, pro- vocada pela simples passagem de nuvem, pode acionar a resposta imediata dos estômatos. Alterações na quantidade de radiação fotossinteticamente ativa disponível, ou no déficit de pressão de vapor, também são prontamente sentidas pela vegetação (Costa et al., 2003b).

Para Bonato et al. (1998) o excesso de chuva satura o solo, eliminando os espaços de ar existentes, dificultando o bom funcionamento das raízes, por outro lado, quando há ausência de chuvas durante longos períodos, a disponibilidade de água para as raízes fica limitada, prejudicando o trabalho de absorção e afetando o transporte de nutrientes para todas as partes do vegetal comprometendo, desta forma, o seu desenvolvimento, ou seja, os eventos extremos (cheia ou seca) são quase sempre danosos aos vegetais. De maneira geral, as plantas absorvem efetivamente pouco da radiação fotossinteticamente ativa (RFA) nos processos fisiológicos, enquanto grande parte é transmitida como aquecimento.

O objetivo do presente trabalho foi determinar a transpiração de exemplares de grande porte de Matá-matá branco (ESchweilera coriacea) na FLONA de Caxiuanã, pelas medidas de fluxo de seiva obtidas com a aplicação do método do balanço de calor no tronco do vegetal, após um longo período de seca provocada artificialmente, a partir do uso de cobertura plástica numa parcela da área experimental.

\section{MATERIAL E MÉTODOS}

\section{Experimento ESECAFLOR}

O Experimento 'O Impacto da Seca Prolongada nos Fluxos de Água e Dióxido de Carbono em uma Floresta Tropical Amazônica' ESECAFLOR consiste na criação, provocada artificialmente, de um período de estiagem na floresta tropical úmida, objetivando avaliar os impactos da seca nos fluxos de água e dióxido de carbono, neste ecossistema; objetiva também analisar a influência desta exclusão de água no solo, sobre o ciclo da floresta e as alterações provocadas pelo evento de seca (Meir et al., 2003).

A estrutura física do ESECAFLOR se constitui de duas parcelas (A e B) de 1 ha cada uma, localizadas a cerca de 800 m de distância da sede da Estação Científica Ferreira Pena (ECFPn). A parcela A foi usada como referência para os experimentos realizados na parcela $\mathrm{B}$, na qual se fez a exclusão de aproximadamente $98 \%$ da água da chuva; ressalta-se haver uma distância de $20 \mathrm{~m}$ entre as parcelas. Essas áreas foram delimitadas por trincheiras de $0,5 \mathrm{~m}$ de largura, cavadas com profundidade variando de 0,5 a $1,5 \mathrm{~m}$ para facilitar a drenagem da chuva. A trincheira da parcela B foi revestida com lona plástica preta, para minimizar a infiltração lateral e, na parcela A, não foi instalado este revestimento. Para cobertura da parcela $\mathrm{B}$ foram utilizados aproximadamente 5000 painéis, com estrutura de madeira e revestimento plástico transparente, instalados a uma altura variando de 1,5 a $4 \mathrm{~m}$ acima do solo. Esses painéis têm largura em torno de $0,5 \mathrm{~m}$, e comprimento aproximado de $3 \mathrm{~m}$, oscilando de acordo com o local, permitindo também a passagem, entre eles, da vegetação de subdossel e piso. Nesta parcela B construíram-se 20 calhas de 
madeira com 0,3 x 0,3 $\mathrm{m}$ de largura e profundidade, que têm $100 \mathrm{~m}$ de comprimento e foram dispostas paralelamente a uma distância de $5 \mathrm{~m}$, com altura em torno dos $4 \mathrm{~m}$ na parte central da parcela e $1,5 \mathrm{~m}$ nas laterais.

Para evitar a infiltração da água interceptada pelos painéis, as calhas também foram revestidas com o mesmo material plástico dos painéis, o que facilitou o escorrimento da água para as trincheiras laterais que direcionam esses fluxos para pontos de descarga distantes da parcela, para dificultar o retorno da água à parcela $\mathrm{B}$. A cobertura da parcela era mantida permanentemente limpa, com substituição dos painéis com a estrutura ou o plástico danificado pela queda de galhos, frutos e, eventualmente, árvores. Utilizou-se, em cada uma das parcelas, uma torre de ferro galvanizado com dimensões de 1,2 × 2,5 x 30 m de largura, comprimento e altura, respectivamente, sustentada por cabos-de-aço estendidos de diferentes alturas até o solo; referidas torres possuíam plataformas e escadas que permitiam fácil acesso às árvores.

\section{Localização}

O sítio experimental do LBA está localizado na Estação Científica Ferreira Pena (ECFPn) (1' 42’30” S; 51 3 31'45" W; $62 \mathrm{~m}$ de altitude) pertencente ao Museu Paraense Emílio Goeldi. A área da ECFPn é de 33.000 ha, localizada dentro da FLONA de Caxiuanã, uma reserva com 330000 ha de floresta primária de terra-firme que ainda preserva as características no município de Melgaço, distante cerca de $400 \mathrm{~km}$ a oeste de Belém.

Lisboa (1997) relatou a FLONA de Caxiuanã como possuidora de ecossistemas de terra-firme, várzea e de igapó e manchas de savana e vegetação secundária. Os rios são de águas negras, ricas em plantas aquáticas. Os limites geográficos são, ao norte, o divisor de águas entre as bacias do Rio Caxiuanã e do Rio Amazonas; ao sul, o Rio Caxiuanã e igarapé Laranjal e, a oeste, o igarapé Grande.

\section{Vegetação}

A FLONA de Caxiuanã agrega ecossistemas riquíssimos em espécies vegetais, o que a caracteriza como uma zona que abrange vários ambientes, dentre eles a floresta densa de terrafirme e igapó (Almeida et al., 1993).

Para Viana et al. (2003) a floresta de terra-firme ocupa cerca de $85 \%$ da área em que foi implantada a ECFPn, a arquitetura florestal se constitui de árvores emergentes (40 a $50 \mathrm{~m}$ ), dossel (30 a $35 \mathrm{~m})$, subdossel (20 a $25 \mathrm{~m})$ e piso $(5 \mathrm{~m})$; a diversidade varia entre 150 a 160 espécies ha-1, a densidade de indivíduos é de 450 a 550 árvores ha ${ }^{-1}$, e a biomassa acima do solo é de $200 \mathrm{~m}^{3} \mathrm{ha}^{-1}$ As espécies predominantes na floresta de terra-firme são: Eschweilera coriacea (Ap. Dec.) Martius ex Berg (Lecythidaceae) ou Matá-matá branco, Voucapoua americana Aublet (Caesalpiniaceae) ou Acapu e Protium pallidum Cuatrec (Burseraceae) ou Breu Branco.

Silva et al. (2003) descreveram essa floresta tropical úmida de terra-firme como apresentando dossel bastante fechado, permitindo pouca penetração dos raios solares, que quase nunca atingem o solo. $\mathrm{O}$ índice de área foliar (IAF) se situa entre 5-6. Até 2003 foram registradas, para a ECFPn, 1054 espécies pertencentes a 393 gêneros e 102 famílias. As espécies arbóreas predominam na ECFPn com 663 spp (62,9\%).

\section{Solo}

Os solos de Caxiuanã vão de bem drenados a moderadamente drenados e pobres em nutrientes; têm cor bruno amarelo-escuro (10YR, 3/6) a vermelho amarelado (7,5YR, 6/6). A base do perfil constitui-se de um horizonte de areia e/ou argila caulinítica, intercalada com um horizonte laterítico. $\mathrm{O} \mathrm{pH}$ varia de 3,5 muito ácido, a 5,5 moderadamente ácido (Ruivo et al., 2002).

A região apresenta relevo relativamente plano que sustenta um Latossolo Amarelo, profundo e de origem terciária, tendo como características marcantes a acidez, solos argilo-arenosos e bastante vulneráveis à erosão laminar. São também encontrados outros tipos, como Plintossolos e Terra Preta arqueológica (Kern, 1996).

\section{Clima}

As informações meteorológicas foram obtidas por uma estação automática e armazenadas em datalogger 21X (Campbell Scintific, USA) instalados no topo da torre de alumínio com 1,1 x 2,2 x 52 m de largura, comprimento e altura, respectivamente (SGB, Edinburgh, UK), sustentada por cabos-deaço estendidos em diversas direções e alturas (Costa et al., 2004). Esta torre foi construída a cerca de $2 \mathrm{~km}$ de distância ao norte do experimento de exclusão de água da chuva (ESECAFLOR). Entre 2000 e 2003, na FLONA de Caxiuanã a média anual de chuva foi de $1865 \mathrm{~mm}$ e a temperatura média do ar registrada foi de $26,3{ }^{\circ} \mathrm{C}$. Valores de temperatura do ar e precipitação anual são apresentados na Tabela 1 .

Tabela 1. Valores anuais de precipitação e temperatura máxima, média e mínima do ar para Caxiuanã, Pará, no período de 2000 a 2003

\begin{tabular}{lrrrrr}
\hline \multicolumn{1}{c}{ Ano } & $\mathbf{2 0 0 0}$ & $\mathbf{2 0 0 1}$ & $\mathbf{2 0 0 2}$ & $\mathbf{2 0 0 3}$ & Média \\
Temp. máxima $\left({ }^{\circ} \mathrm{C}\right)$ & 34,0 & 37,1 & 35,2 & 34,3 & 35,1 \\
Temp. média $\left({ }^{\circ} \mathrm{C}\right)$ & 25,8 & 26,9 & 26,3 & 26,1 & 26,3 \\
Temp. mínima $\left({ }^{\circ} \mathrm{C}\right)$ & 20,9 & 21,6 & 20,5 & 20,2 & 20,8 \\
Precipitação $(\mathrm{mm})$ & 1518,7 & 1697,9 & 2192,8 & 2049,5 & 1865,0 \\
\hline
\end{tabular}

\section{Fluxo de seiva}

Para as medidas de fluxo de seiva foi aplicado o método de Balanço de Calor no Tronco (THB - Trunk Heat Balance), através do sistema Sap Flow meter P4.1, (EMS, Brno, Republica Checa). A técnica se caracteriza pelo aquecimento de parte do tronco dos vegetais por uma corrente elétrica $(0,6-$ $1 \mathrm{~W})$; o aquecimento é proporcional à energia que passa por eletrodos de aço inseridos no tecido vegetal, sendo o calor liberado uniformemente dentro do xilema. A energia aplicada (P) é dividida entre a perda de calor por condutividade e o aquecimento da água, que passa através da área aquecida. Esta energia é diretamente proporcional ao fluxo de seiva e a diferença de temperatura (dT) é inversamente proporcional ao fluxo, as quais podem ser mantidas constantes pelo circuito eletrônico, enquanto as outras variáveis são registradas (Kuèera, 1998; Ėermák et al., 2004). O método calcula o balanço de calor com base na razão entre a energia $\mathrm{P}$ e o aumento da temperatura em um espaço do tronco vegetal definido entre os sensores térmicos. Realizaram-se medidas a cada minuto e 
se armazenou a média em intervalos de 15 minutos no módulo interno de memória do sistema, que tem capacidade para até 14 dias de informações quando todos os 12 canais estão em uso. O balanço de calor na área do tronco foi obtido pela Eq. 1.

$$
\mathrm{P}=\mathrm{Q} \cdot \mathrm{dT} \cdot \mathrm{c}_{\mathrm{W}}+\mathrm{dT} \cdot \mathrm{z}
$$

em que

$\mathrm{P}$ - energia para o aquecimento (W),

Q - fluxo de seiva $\left(\mathrm{kg} \mathrm{s}^{-1}\right)$,

dT - diferença de temperatura no ponto de medida $(\mathrm{K})$,

$\mathrm{c}_{\mathrm{w}}$ - calor específico da água (4 186,8 $\mathrm{J} \mathrm{kg}^{-1} \mathrm{~K}^{-1}$ )

$\mathrm{Z}$ - coeficiente de dissipação do calor no ponto de medida $\left(\mathrm{W} \mathrm{K}^{-1}\right)$

A quantidade de água que passa no ponto de medida no tronco da arvore é calculada usando-se o valor da temperatura no xilema e a energia aplicada no aquecimento. A escolha do ponto de medida no tronco é fundamental para a qualidade das informações. A homogeneidade do tronco e a ausência de injúrias (nós ou cortes), que poderiam prejudicar o transporte da água no xilema, ajudam na qualidade das medidas. A altura a serem instalados os eletrodos também é significativa, um gradiente térmico ocorre próximo à superfície do solo, o que pode interferir nas medições; em geral, escolhe-se uma altura próxima a 1,3 m (altura do peito usada em inventários florestais). Inseriram-se, no ponto de medida escolhido, paralelamente, 5 eletrodos de aço inoxidável que atingem o xilema e são aquecidos pela corrente elétrica. As peças metálicas têm $1 \mathrm{~mm}$ de espessura, $25 \mathrm{~mm}$ de largura e comprimento variando entre 60 e $80 \mathrm{~mm}$, dependendo das dimensões da árvore inserida com uma distância lateral de 20 $\mathrm{mm}$ entre elas. $\mathrm{O}$ eletrodo central é posicionado em direção radial ao tronco. Dois pares de agulha (termopares de cobreconstantan, $\mathrm{Cu}-\mathrm{Cst}$ ) com 50 x $1 \mathrm{~mm}$ de comprimento e diâmetro, respectivamente, foram inseridos em orifícios do mesmo diâmetro das agulhas feitos com furadeira elétrica, sendo que duas agulhas são instaladas na parte superior, na altura dos eletrodos, próximo ao eletrodo central, e as outras duas são colocadas $120 \mathrm{~mm}$ abaixo, na mesma direção das agulhas acima. A distância lateral entre duas agulhas é de $20 \mathrm{~mm}$. Em cada par desses sensores térmicos a agulha do lado esquerdo é inserida a uma profundidade maior que a do lado direito. As diferenças são fixadas para cada tamanho de eletrodo, 13, 17 e $23 \mathrm{~mm}$ para os eletrodos de 60,70 e $80 \mathrm{~mm}$, respectivamente. As profundidades que as agulhas atingirão são determinadas de acordo com as características da planta, como diâmetro e espessura da casca e do floema.

Após a instalação, os sensores foram protegidos da ação do clima, pela utilização de finas capas de alumínio flexível revestidas de esponja e cobertores de plástico transparente, vedados na parte superior com fita adesiva e graxa natural, evitando a infiltração da água escorrida pelo tronco, que poderia causar curtos-circuitos.

Nos períodos de 3 a 16 de dezembro de 2000, antes do fechamento da cobertura plástica na parcela $\mathrm{B}$ e também entre 12 e 26 de janeiro de 2003, após 13 meses de influência do estresse hídrico provocado pela exclusão da chuva, foram monitorados dois exemplares de Eschweilera coriacea (Matámatá branco) identificados como A237, localizado na parcela A (controle) e B381 na parcela B (seca). As características dessas plantas são apresentadas na Tabela 2. O intervalo de mais de um ano, ocorrido entre o fechamento da cobertura na parcela B (em dezembro de 2001) e a repetição das medidas de fluxo (em janeiro de 2003) foi necessário para uma definição melhor dos efeitos sentidos pelo ecossistema, devido ao evento da seca provocada. Nos 14 dias de medidas em dezembro de 2000 as plantas estavam sob as mesmas condições de umidade no solo; entretanto, nos 14 dias, em janeiro de 2003, a parcela B apresentava redução no teor de água no solo disponível à planta.

Tabela 2. Dimensões dos exemplares de E. coriacea monitorados no ESECAFLOR

\begin{tabular}{|c|c|c|c|c|c|c|c|}
\hline \multirow{2}{*}{ ID } & & Altura & DAP & & PM & \multirow{2}{*}{\multicolumn{2}{|c|}{$\frac{\text { ACPS }}{\mathrm{m}^{2}}$}} \\
\hline & & & m & & & & \\
\hline A (237) & 27 & & 0,337 & 1,06 & & 187 & \\
\hline B (381) & 33 & $+22 \%$ * & 0,315 & 0,99 & $-6,6 \%$ * & 123 & $-34 \%$ * \\
\hline
\end{tabular}

ACPS - Área plana da Copa da ánore Projetada no Solo

CPM- Circunferéncia do tronco no Ponto de Medida

DPM- Circunterènclia do tronco no Ponto de Me

*Valores (\%) sáo diferenças da árvore B381 em relação a A237

\section{Conteúdo de água no solo}

Meir et al. (2002) descreveram que em cada parcela do ESECAFLOR foram abertas quatro trincheiras de amostragem (A1, A2, A3, A4 e B1, B2, B3, B4) com as dimensões de 1 x 2 $\mathrm{x} 5 \mathrm{~m}$ de largura, comprimento e profundidade, respectivamente, para se avaliar as características do solo anteriores à exclusão de água na floresta e o monitoramento do conteúdo de água no solo. Essas trincheiras eram cobertas por tampas de madeira revestidas com plástico transparente, igual ao utitizado na cobertura das parcelas, minimizando ainda mais a possibilidade de entrada de chuva no local.

Para as medições foi utilizado o sistema de TDR (TimeDomain Reflectometer) com um osciloscópio Tektronic 1502B/C e o software WATTDR 3.11 (Waterloo Centre for Groundwater Research). Os sensores de umidade foram instalados nas duas paredes menores da trincheira, a cada metro de profundidade, desde a superfície até $5 \mathrm{~m}$. As medidas de TDR foram realizadas quinzenalmente.

\section{RESULTADOS E DISCUSSÃO}

Apresentar-se-á breve análise relacionada a alguns fatores meteorológicos que influenciaram nas respostas transpirativas dos vegetais, durante os dois períodos de estudo no experimento ESECAFLOR, mostrados neste trabalho.

No período de 2000 a 2003, o mês mais chuvoso foi março, com média de $295 \mathrm{~mm}$, enquanto o mais seco foi agosto, com chuva de $45 \mathrm{~mm}$ em média. Aproximadamente, $73 \%$ das chuvas ocorreram entre janeiro e junho, período em que as médias para os meses de janeiro e dezembro foram de 242 e 150 $\mathrm{mm}$, respectivamente. Em dezembro de 2000 foram registrados apenas $87 \mathrm{~mm}$ de chuva, $58 \%$ da média do período, enquanto 
em janeiro de 2003 foram $187 \mathrm{~mm}$, ou seja, 77\% da média do período.

Para o período de 3 a 16 de dezembro de 2000 houve ocorrência de chuvas em 10 dos 14 dias estudados, com um volume total de $65 \mathrm{~mm}$, sendo o máximo diário de $19 \mathrm{~mm}$ no dia 15 . No período de 12 a 25 de janeiro de 2003, o total precipitado foi de $92 \mathrm{~mm}$, com máximo diário de $22 \mathrm{~mm}$, no dia 24 . Neste mesmo período choveu em todos os 14 dias estudados (Tabela 3 ).

Ambos os períodos da pesquisa estão inseridos na época chuvosa da região de Caxiuanã; desta maneira, a disponibilidade de água para as raízes estava satisfatória na parcela $\mathrm{A}$, tanto em dezembro de 2000 como em janeiro de 2003. Considerando-se que na parcela B mais de $90 \%$ das chuvas foram interceptados pelos painéis plásticos durante o ano de 2002 , esta área apresentou menor diponibilidade de água no solo em janeiro de 2003, para suprir as necessidades hídricas da vegetação local.

A floresta funciona como controlador das variações aerotérmicas, não permitindo oscilação acentuada durante o dia. A vegetação densa reflete, para a atmosfera, uma parte da radiação solar, que chega ao topo do dossel; outra parte da energia disponível é utilizada nos processos de fotossíntese realizados pelas plantas, deixando apenas uma pequena parte da luz solar penetrar entre as diversas camadas de folhagem e atingir o solo porém, após aquecer a superfície, parte desta energia fica armazenada na camada de ar compreendida entre o solo e a parte inferior do dossel, propiciando um aquecimento constante e regular.

A temperatura do ar alcançou valores máximos, normalmente acima dos $32^{\circ} \mathrm{C}$, em geral em torno das $13 \mathrm{~h}$, principalmente nos meses de menor pluviosidade (julho a dezembro). A temperatura máxima absoluta foi de $37,1^{\circ} \mathrm{C}$ registrada em dezembro de 2000; em média, de 2000 a 2003, o mês mais quente foi dezembro registrando-se $33,7^{\circ} \mathrm{C}$ e as temperaturas mínimas ocorreram logo ao amanhecer, alcançando valores em torno de $21^{\circ} \mathrm{C}$. A temperatura mínima absoluta no período foi de 20,2 ${ }^{\circ} \mathrm{C}$ em setembro de 2003; na média, o mês mais frio foi setembro, com $21,5^{\circ} \mathrm{C}$.

No período entre 2000 e 2003 as temperaturas médias nos meses de janeiro e dezembro foram de 25,6 e $26,9^{\circ} \mathrm{C}$, res- pectivamente, enquanto as médias registradas em dezembro de 2000 e janeiro de 2003 foram de 26,3 e $25,2^{\circ} \mathrm{C}$, respectivamente. As diferenças observadas nas médias dos meses de estudo em relação às médias do período total foram de apenas $2 \%$, aproximadamente.

Os períodos estudados apresentaram muita nebulosidade (época chuvosa) reduzindo, desta forma, a quantidade de energia radiante que atingiu o solo; mesmo assim, a área experimental, por estar localizada na região equatorial e sujeita a nebulosidade cumuliforme, registrou picos horários da radiação de ondas curtas incidente (ROC) acima de $980 \mathrm{Wm}^{-2}$ em dezembro de 2000 e de até $950 \mathrm{Wm}^{-2}$ em janeiro de 2003.

Em dezembro de 2000, para os valores de ROC integrados diariamente, das 07 às $18 \mathrm{~h}$, o máximo e o mínimo foram de 20,6 e 10,0 $\mathrm{MJ} \mathrm{m}^{-2}$, nos dias 5 e 16, respectivamente, enquanto no período de janeiro de 2003 foram de 20,3 e 9,9 $\mathrm{MJ} \mathrm{m}^{-2}$ nos dias 25 e 22 , respectivamente (Tabela 3 ). A quantidade de energia radiante disponível nos dois períodos foi similar e as pequenas diferenças observadas estão relacionadas com o horário de ocorrência de cobertura de nuvens e das chuvas.

A radiação fotossinteticamente ativa (RFA) disponível atingiu valores superiores a $1600 \mathrm{mmol} \mathrm{m}^{-2} \mathrm{~s}^{-1}$, em dezembro de 2000 e a $1700 \mathrm{mmol} \mathrm{m}^{-2} \mathrm{~s}^{-1}$, em janeiro de 2003 .

Durante os 14 dias analisados em dezembro de 2000, os valores máximo e mínimo diários de RFA integrados das 07 às $18 \mathrm{~h}$, foram de 34,3 e $18,1 \mathrm{~mol} \mathrm{~m}^{-2}$ nos dias 05 e 16 , respectivamente, enquanto no período de janeiro de 2003 os valores máximo e mínimo foram 37,7 e 24,0 $\mathrm{mol} \mathrm{m}^{-2}$ nos dias 25 e 22, respectivamente (Tabela 3 ). Como ocorre continuamente na Amazônia, também durante os períodos estudados as plantas receberam grande suprimento de RFA.

\section{Conteúdo de água no solo}

O solo da ECFPn apresentou uma particularidade com relação a abertura das trincheiras de $5 \mathrm{~m}$ de profundidade nas parcelas. Em torno dos $3 \mathrm{~m}$ de profundidade, dá-se ocorrência de uma camada de solo laterítico, com aproximadamente $0,3 \mathrm{~m}$ de espessura; esta presença rochosa dificulta a penetração das raízes para as camadas mais profundas do solo. São exibidos, na Figura 1, os teores de água no solo, a partir de novembro de 2000; em meados de dezembro de 2001 iniciouse, na parcela B, o processo de exclusão da água da chuva.

Tabela 3. Valores diários da precipitação pluvial (Prp); Radiação de ondas curtas (ROC); Radiação Fotossinteticamente Ativa (RFA) e Temperatura média do $\operatorname{Ar}$ (Tar). ROC e RFA foram integrados das 07 às $18 \mathrm{~h}$

\begin{tabular}{|c|c|c|c|c|c|c|c|c|c|c|c|c|c|c|c|}
\hline \multicolumn{16}{|c|}{ Dezembro de 2000} \\
\hline Dias & 3 & 4 & 5 & 6 & 7 & 8 & 9 & 10 & 11 & 12 & 13 & 14 & 15 & 16 & Média \\
\hline Prp (mm) & 0,0 & 1,6 & 4,2 & 2,2 & 0,2 & 12,0 & 0,0 & 0,0 & 0,0 & 1,4 & 8,0 & 1,4 & 19,0 & 10,2 & 4,3 \\
\hline $\mathrm{ROC}\left(\mathrm{MJ} \mathrm{m}^{-2}\right)$ & 19,5 & 13,5 & 20,6 & 16,3 & 19,7 & 19,4 & 17,1 & 16,8 & 18,9 & 15,6 & 11,7 & 17,0 & 11,8 & 10,0 & 16,0 \\
\hline RFA $\left(\mathrm{mol} \mathrm{m} \mathrm{m}^{-2}\right)$ & 32,3 & 22,4 & 34,3 & 26,9 & 31,6 & 31,4 & 28,9 & 28,3 & 29,4 & 26,0 & 20,2 & 30,1 & 21,3 & 18,1 & 26,8 \\
\hline $\operatorname{Tar}\left({ }^{\circ} \mathrm{C}\right)$ & 26,9 & 26,4 & 26,4 & 26,1 & 26,9 & 26,8 & 26,7 & 27,3 & 27,4 & 26,2 & 24,7 & 25,5 & 24,0 & 24,5 & 26,1 \\
\hline \multicolumn{16}{|c|}{ Janeiro de 2003} \\
\hline Dias & 12 & 13 & 14 & 15 & 16 & 17 & 18 & 19 & 20 & 21 & 22 & 23 & 24 & 25 & Média \\
\hline Prp (mm) & 3,6 & 4,2 & 8,2 & 5,6 & 7,2 & 7,0 & 6,6 & 5,0 & 5,4 & 0,2 & 1,0 & 0,8 & 21,8 & 0,2 & 5,5 \\
\hline $\mathrm{ROC}\left(\mathrm{MJ} \mathrm{m}^{-2}\right)$ & 16,0 & 13,2 & 12,8 & 12,8 & 10,7 & 14,2 & 14,9 & 15,9 & 15,6 & 13,1 & 9,9 & 15,1 & 14,9 & 20,3 & 14,2 \\
\hline RFA $\left(\mathrm{mol} \mathrm{m}{ }^{-2}\right)$ & 35,8 & 28,5 & 29,2 & 29,3 & 24,5 & 32,6 & 34,7 & 36,9 & 36,7 & 30,6 & 24,0 & 30,9 & 28,6 & 37,7 & 31,4 \\
\hline $\operatorname{Tar}\left({ }^{\circ} \mathrm{C}\right)$ & 26,1 & 26,3 & 25,4 & 25,3 & 24,9 & 25,8 & 25,4 & 25,3 & 24,4 & 25,0 & 24,6 & 25,3 & 25,2 & 26,0 & 25,3 \\
\hline
\end{tabular}


A redução do volume de água que atingiu o solo nesta área provocou diminuição acentuada nos valores medidos de umidade do solo, já observada a partir de fevereiro de 2002 (Figura 1A); este estresse hídrico forçado, ao qual foram submetidas as plantas da parcela coberta (B), provocou alterações nas respostas transpiratórias das árvores de E. coriacea monitoradas. As medições realizadas no solo mostraram grande variação no conteúdo de água entre as parcelas. Comparando-se os volumes, concluiu-se que a maior diferença foi de $-33 \%$ de água na parcela B no mês de junho de 2002, final do período chuvoso (Figura 1B), ano em que não se deu, durante a época das chuvas, reposição dos estoques normais da água no solo desta parcela (conteúdo pré-cobertura da área). A disponibilidade hídrica para as raízes foi reduzida alterando, concomitantemente, o volume de água transportado pelos vegetais para a atmosfera.

No período de julho a dezembro de 2002, as diferenças no conteúdo de água entre as parcelas diminuíram, mais pelo fato de também haver redução no teor de umidade do solo da parcela A do que por uma elevação no conteúdo da parcela B. Com o retorno do período chuvoso, no início de 2003, as diferenças voltaram a crescer, mostrando a eficiência do trabalho estrutural realizado na cobertura do Experimento ESECAFLOR, que permitiu avaliar-se o impacto causado pelo período acentuado de seca na região.

O conteúdo de água no solo, armazenado na camada da superfície até $3 \mathrm{~m}$, superior ao horizonte laterítico, durante o ano de 2002 (Figura 1A), oscilou próximo de $600 \mathrm{~mm}$ na parcela $\mathrm{B}$, nos meses de janeiro a junho, sendo o máximo registrado em abril, com $602 \mathrm{~mm}$. Em janeiro de 2003 mediu-se o nível mais baixo de água no solo com $452 \mathrm{~mm}$ e, como previsto, a sazonalidade natural da umidade do solo foi alterada na parcela B; em 13 de janeiro de 2003, a parcela B atingiu $-30,5 \%$ de água no solo, em relação a 15 de dezembro de 2000; por outro lado, na parcela de controle (A) a sazonalidade foi mantida, sendo registrados os valores máximo (março) e mínimo (outubro) de 776 e $530 \mathrm{~mm}$, respectivamente; enfim, em 13 de janeiro de 2003 a parcela A registrou redução de apenas $9,1 \%$ de água no solo, em relação a 15 de dezembro de 2000.

\section{Fluxo de seiva}

As medidas de fluxo de seiva realizadas em duas arvores de Eschweilera coriacea (Matá-matá branco) na área experimental do ESECAFLOR, no período chuvoso da região caracterizam, de forma bem definida, os ciclos transpirativos desta espécie tropical. Em dezembro de 2000, ambas as arvores estavam em condições climáticas semelhantes; esta similaridade de ambiente está representada nas respostas fisiológicas descritas na Figura 2. No A237, monitorado na parcela de referência (A), entre 03 e 16 de dezembro de 2000 (Figura 2A) os fluxos atingiram valores acima de $25 \mathrm{~kg} \mathrm{arv}^{-1} \mathrm{~h}^{-1}$ (kilograma de seiva pela circunferência do tronco da árvore por hora que, a partir daqui, será descrito apenas como $\mathrm{kg} \mathrm{h}^{-1}$ ), nos dias 3, 8 e 9, sendo registrado, no dia 9, o máximo de 27 $\mathrm{kg} \mathrm{h}^{-1}$, período em que o fluxo médio foi, em A237, de 4,0 kg $\mathrm{h}^{-1}$. Para a árvore B381, localizada na parcela B que foi submetida a exclusão da chuva a partir de dezembro de 2001, os

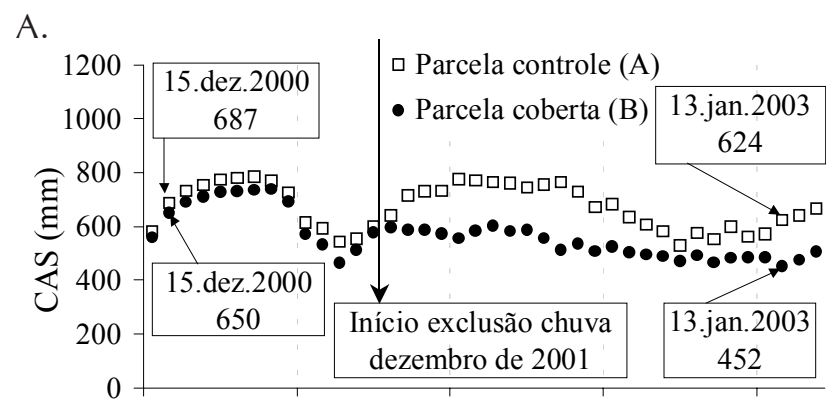

B.

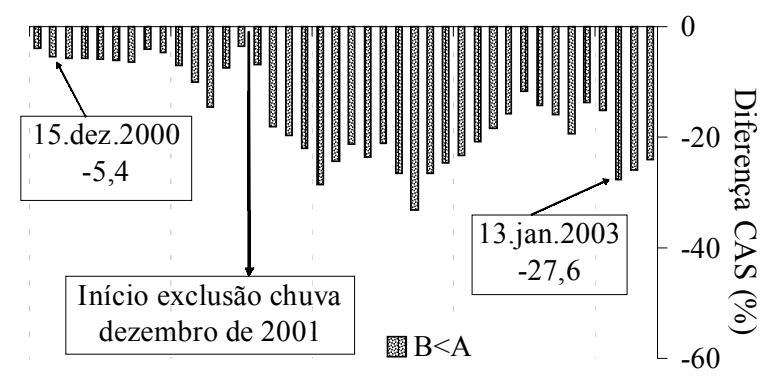

29-Nov-00 17-Aug-01 24-Mar-02 10-Aug-02 13-Dec-02

Figura 1. Conteúdo de água no solo - CAS (mm) (1A) e diferenças (\%) (1B), na camada de 0 a $3 \mathrm{~m}$, nas parcelas do ESECAFLOR. O ciclo sazonal foi alterado na parcela B

fluxos máximos alcançaram valores acima de $38 \mathrm{~kg} \mathrm{~h}^{-1}$ nos dias 3, 6 e 9 (Figura 2B); na média, o fluxo foi de 7,3 $\mathrm{kg} \mathrm{h}^{-1}$, cuja diferença foi creditada somente às características de cada arvore, como altura total, diâmetro do tronco, área da copa e maior exposição à luz solar, já que estão em áreas fisicamente similares.

Em dezembro de 2001, o trabalho de cobertura da parcela B foi finalizado e se iniciaram, daí, as medidas sob o processo de exclusão de mais de $90 \%$ da água das chuvas que ocorreram na região. No começo do período chuvoso de 2002, o solo já apresentava redução no teor de água porém ainda continha grande volume armazenado; no entanto, com a cobertura plástica na parcela $\mathrm{B}$ o conteúdo de água começou a não mais ser reposto integralmente reduzindo, desta maneira, a disponibilidade hídrica às plantas. Em meados de 2002, com a chegada da época menos chuvosa, a diferença já era claramente visível, como foi determinado pelas medidas de TDR (Figura 1B), sendo que o solo da parcela B chegou a apresentar, na camada da superfície até os $3 \mathrm{~m}$ de profundidade, cerca de dois terços do teor de umidade registrado na parcela A.

Nas medidas de fluxo de seiva da E. Coriacea, realizadas entre 12 e 25 de janeiro de 2003, os efeitos da seca forçada (13 meses) a que foi submetido o exemplar da parcela B, foram bastante evidentes. A planta A237, na parcela A continuou a registrar valores de fluxo da mesma magnitude dos obtidos em dezembro de 2000, até mais elevados, atingindo os $37 \mathrm{~kg} \mathrm{~h}^{-1}$, nos dias 18 e 25 (Figura 2C) período em que o fluxo médio em A237 foi de $6,1 \mathrm{~kg} \mathrm{~h}^{-1}$; enquanto isso, a árvore da parcela $B$ teve os fluxos reduzidos de maneira acentuada (Figura 2D). O exemplar B381, sob estresse hídrico, transpirou a taxas em torno de $10 \mathrm{~kg} \mathrm{~h}^{-1}$, uma redução para cerca 
A.

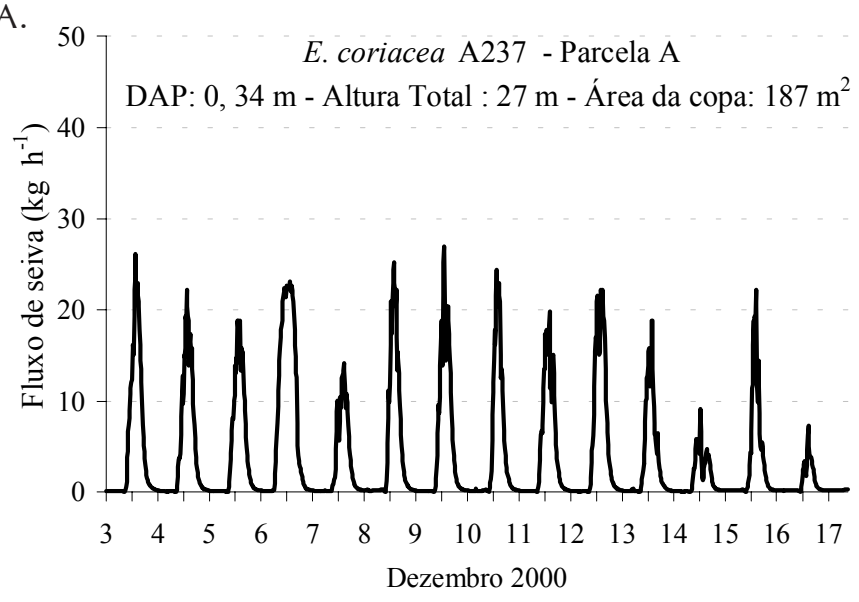

C.

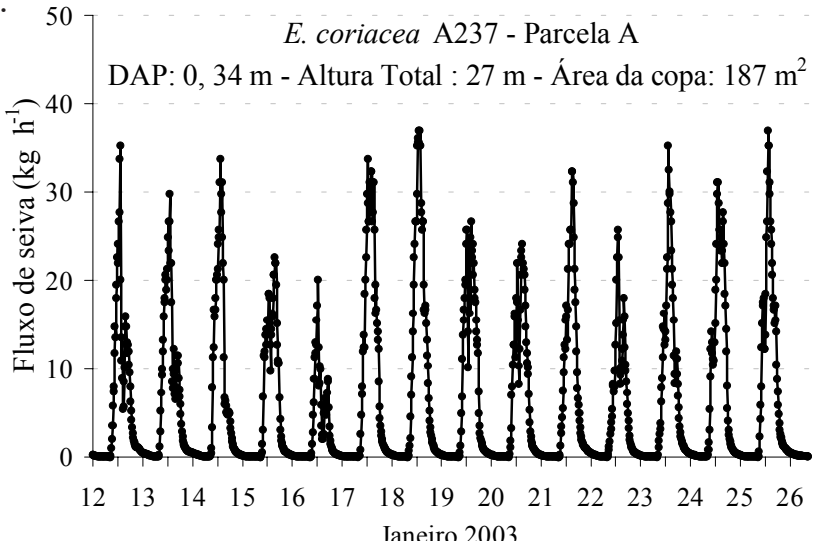
E. coriaceà B381 - Parcela B

DAP: $0,32 \mathrm{~m}$ - Altura Total : $33 \mathrm{~m}$ - Área da copa: $123 \mathrm{~m}^{2}$

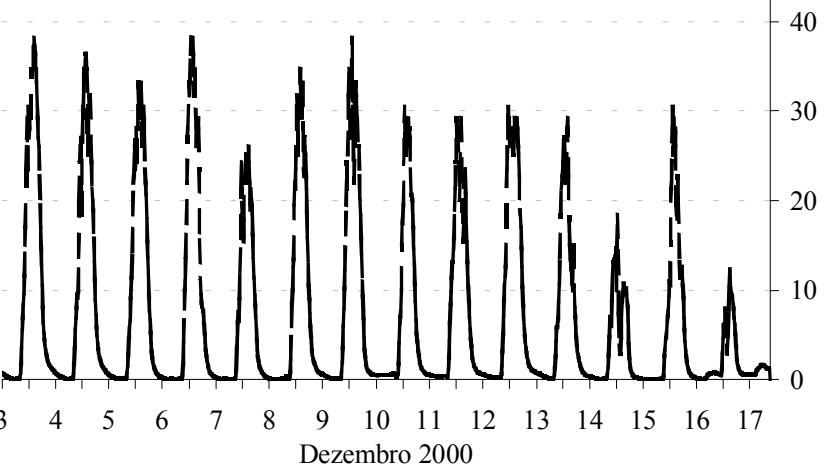

D.

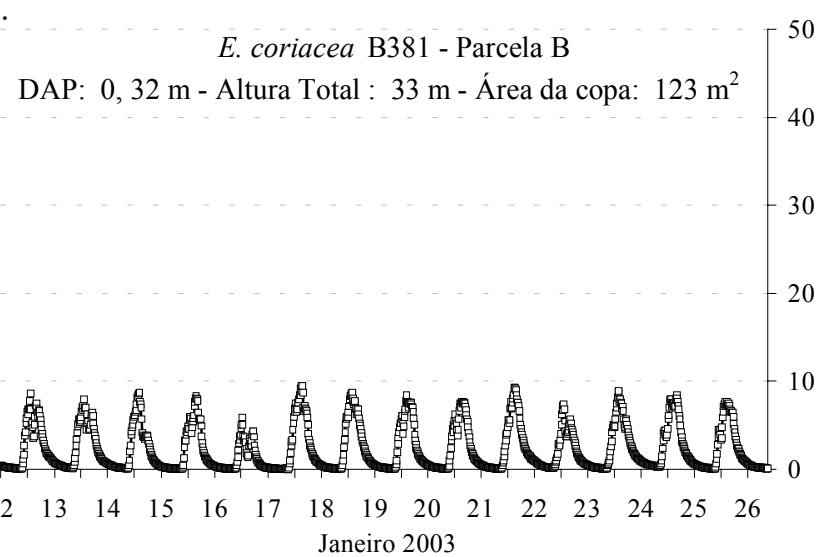

Figura 2. Fluxo de seiva $\left(\mathrm{kg} \mathrm{h}^{-1}\right)$ nos dois exemplares de $E$. Coriacea. As Figuras (2A) e (2B) referem-se ao período de 3 a 17 de dezembro de 2000 , enquanto as Figuras (2C) e (2D) entre 12 e 25 de janeiro de 2003. Parcela A (controle), Parcela B (submetida ao estresse)

Tabela 4. Valores diários da transpiração (kg) de E. Coriacea nas árvores A237 (referência) e B381 submetida ao estresse hídrico a partir de dezembro de 2001

\begin{tabular}{|c|c|c|c|c|c|c|c|c|c|c|c|c|c|c|c|}
\hline \multirow[b]{2}{*}{ Dias } & \multicolumn{14}{|c|}{ Dezembro de 2000} & \multirow[b]{2}{*}{ Médie } \\
\hline & 3 & 4 & 5 & 6 & 7 & 8 & 9 & 10 & 11 & 12 & 13 & 14 & 15 & 16 & \\
\hline A237 & 122 & 107 & 100 & 191 & 72 & 113 & 120 & 102 & 100 & 123 & 80 & 38 & 78 & 24 & 98 \\
\hline B381 & 248 & 227 & 215 & 226 & 155 & 201 & 225 & 167 & 179 & 216 & 161 & 90 & 143 & 54 & 179 \\
\hline \multicolumn{16}{|c|}{ Janeiro de 2003} \\
\hline Dias & 12 & 13 & 14 & 15 & 16 & 17 & 18 & 19 & 20 & 21 & 22 & 23 & 24 & 25 & Média \\
\hline A237 & 141 & 149 & 147 & 128 & 70 & 194 & 205 & 160 & 140 & 156 & 110 & 157 & 174 & 179 & 151 \\
\hline B381 & 60 & 61 & 55 & 52 & 31 & 64 & 63 & 60 & 59 & 64 & 47 & 63 & 67 & 60 & 58 \\
\hline
\end{tabular}

de $32 \%$ dos fluxos medidos em dezembro de 2000. O fluxo médio em B381 foi de apenas 2,3 $\mathrm{kg} \mathrm{h}^{-1}$.

Quando foram integrados diariamente, os resultados dos fluxos de seiva de cada árvore de E. Coriacea monitorada nos dois períodos estudados foram os seguintes: em dezembro de 2000, arvore A237, situado na parcela de referência transportou, à atmosfera, em média $98 \mathrm{~kg}$ de água, alcançando o máximo e o mínimo de 191 e $24 \mathrm{~kg}$ nos dias 6 e 16/12/ 2000 , respectivamente. Como esta árvore não sofreu carência hídrica, em janeiro de 2003 continuou a transpirar com a mesma intensidade, registrando-se, neste período, a média de 151 kg, com o máximo e o mínimo de 205 e $70 \mathrm{~kg}$ nos dias 18 e 16/ 01/2003, respectivamente (Tabela 4).
A arvore B381, submetido ao estresse hídrico forçado pela cobertura plástica na parcela B sofreu, durante o ano de 2002, uma variação acentuada na quantidade de água transpirada. Os valores de fluxo de seiva integrados diariamente foram de maneira significativa superiores no período de dezembro de 2000 anterior à cobertura, em relação a 2003. Com freqüência, variaram acima dos $200 \mathrm{~kg}$ (em 7 dos 14 dias analisados), sendo que a média no período foi de $179 \mathrm{~kg}$; esta árvore atingiu os fluxos máximo e mínimo de 248 e $54 \mathrm{~kg}$, nos dias 3 e $16 / 12 / 2000$, respectivamente; no entanto, em janeiro de 2003, após mais de um ano submetida às restrições de disponibilidade de água, a planta transpirou apenas um terço do volume medido em dezembro de 2000 , em média $58 \mathrm{~kg}$, e com o máximo e o mínimo de 67 e 24 kg nos dias 24 e 16/1/2003, res- 
pectivamente; neste período, a árvore B381 apresentou acentuada queda de folhas e envelhecimento anormal de alguns galhos (mudanças na coloração e maior fragilidade), em diferentes alturas do dossel foliar. Os valores da transpiração diária das árvores monitoradas são apresentados na Tabela 4. Não foi possível determinar exatamente as causas das duas árvores terem registrado os fluxos mínimos nos mesmos dias, para os dois períodos; sugere-se, então, que a combinação de fatores meteorológicos como precipitação, altas nebulosidade e umidade do ar com pouca radiação solar, tenha inibido a atividade transpiratória das plantas naqueles dias.

Considerando-se a Área plana da Copa da árvore Projetada no Solo (ACPS), calculou-se a transpiração por unidade de área, com a divisão da transpiração total diária pela área da copa (Tr/ACPS), em cada exemplar.

Para a árvore A237, com ACPS de $187 \mathrm{~m}^{2}$ (Tabela 1), as médias obtidas nos períodos estudados foram de 0,52 e 0,81 $\mathrm{mm} \mathrm{d}^{-1}$ com os máximos de 1,02 e 1,10 $\mathrm{mm} \mathrm{d}^{-1}$ em 2000 e 2003, respectivamente. Notou-se aumento médio de $55,8 \%$ entre os dois períodos.

$\mathrm{Na}$ árvore B381 com ACPS de $123 \mathrm{~m}^{2}$, as médias foram de 1,46 e $0,47 \mathrm{~mm} \mathrm{~d}^{-1}$, com máximos de 2,02 e $0,55 \mathrm{~mm} \mathrm{~d}^{-1}$ em 2000 e 2003, respectivamente. Como se previa, ocorreu redução média na transpiração, de $67,8 \%$, entre os períodos analisa- dos de 2000 e 2003.

As taxas de evapotranspiração para florestas tropicais são em torno de $4 \mathrm{~mm} \mathrm{~d}^{-1}$, com variações sazonais decorrentes da existência ou não de chuvas (Fisch et al., 1998), porém nesses casos todos os fatores são considerados, por exemplo, a superposição de camadas foliares e a contribuição da superfície. A evapotranspiração da floresta na região Amazônica foi objeto de alguns estudos (Villa Nova et al., 1976, Marques et al., 1980, Shuttleworth et al., 1987). No caso do experimento ESECAFLOR, os resultados consideraram apenas a contribuição de exemplares isolados, ou seja, a água efetivamente transferida pela planta para a atmosfera (transpiração), sem adição do potencial evaporativo do solo.

Analisaram-se, na Figura 3, os ciclos médios diários da radiação fotossinteticamente ativa (RFA) e os fluxos de seiva (FS). No período de 3 a 16 de dezembro de 2000 (3A), os valores de RFA e FS nas plantas A237 e B381 se mantiveram na mesma ordem de grandeza com um volume de água no solo em torno de $650 \mathrm{~mm}$ mas no período de 12 a 25 de janeiro de 2003 (3B) o FS na arvore B381 sofreu intensa redução nos valores registrados, fato indicativo de que, mesmo com grande quantidade de RFA disponível à planta e ainda um teor de água no solo de aproximadamente $450 \mathrm{~mm}$, o estresse hídrico prevaleceu, reduzindo a transpiração.

Conforme a Figura 3, um pequeno atraso na resposta

Tabela 5. Equações das regressão linear e coeficientes de determinação $\left(R^{2}\right)$ entre as médias horárias do fluxo de seiva (FS), radiação fotossinteticamente ativa (RFA) e radiação de ondas curtas (ROC), nos exemplares A237 e B381 de E. Coriacea

\begin{tabular}{|c|c|c|c|c|}
\hline & \multicolumn{2}{|l|}{ A 237} & \multicolumn{2}{|c|}{ B 381} \\
\hline & Equação & $\mathrm{R}^{2}$ & Equação & $\mathbf{R}^{2}$ \\
\hline Radiação & \multicolumn{3}{|c|}{03 a 16 de dezembro de 2000} & \\
\hline RFA & $F S=0,0123 R F A+0,1469$ & 0,8056 & $\mathrm{FS}=0,0211 \mathrm{RFA}+0,6988$ & 0,8212 \\
\hline ROC & $\mathrm{FS}=0,0202 \mathrm{ROC}+0,1943$ & 0,7483 & $\mathrm{FS}=0,0351 \mathrm{R} 0 \mathrm{C}+0,7023$ & 0,7811 \\
\hline \multicolumn{5}{|c|}{12 a 25 de janeiro de 2003} \\
\hline RFA & $\mathrm{FS}=0,0169 \mathrm{RFA}+0,1483$ & 0,9116 & $F S=0,0046 R F A+0,7371$ & 0,7106 \\
\hline ROC & $\mathrm{FS}=0,0374 \mathrm{R} 0 \mathrm{C}+0,1102$ & 0,8725 & $\mathrm{FS}=0,01 \mathrm{ROC}+0,7394$ & 0,67 \\
\hline
\end{tabular}

A.

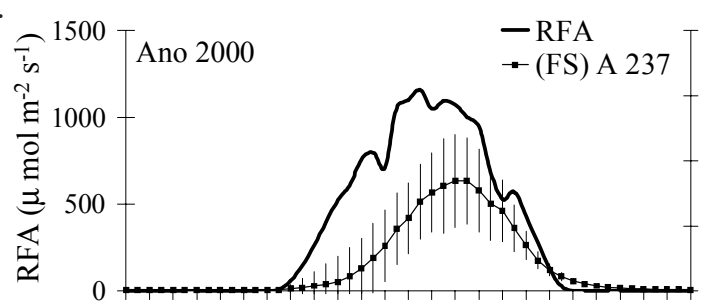

C.

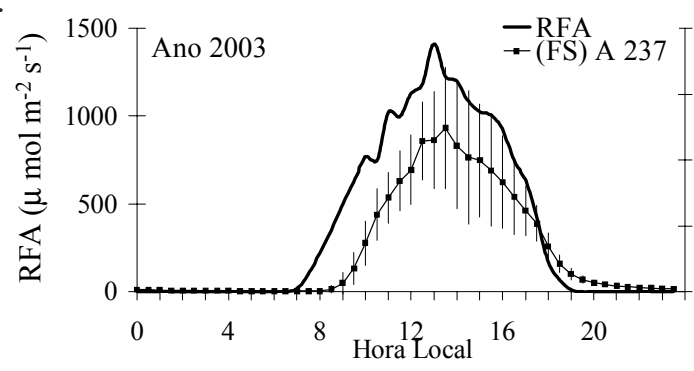

B.

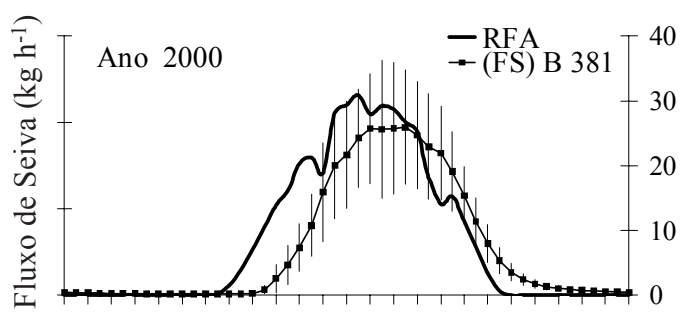

D.

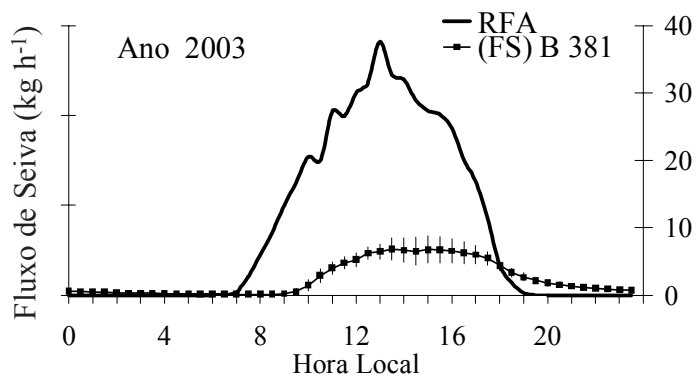

Figura 3. Ciclos médios diários da radiação fotossinteticamente ativa (RFA) $\left(\mathrm{mmol} \mathrm{m}^{-2} \mathrm{~s}^{-1}\right)$ e do fluxo de seiva (FS) $\left(\mathrm{kg} \mathrm{h}^{-1}\right)$, nas árvores A237 e B381 de E. Coriacea As Figuras 3A e 3B são do período de 3 a 17 de dezembro de 2000 e as Figuras 3C e 3D, de 12 a 25 de janeiro 2003. A barra vertical representa o desvio padrão 
transpiratória em relação a RFA mostra, entretanto, que os ciclos são coincidentes. Após o término da incidência de RFA, em torno das $18 \mathrm{~h}$, o FS se estendeu ainda pelas primeiras horas da noite, até atingir a estabilização (fluxo zero), por volta das $3 \mathrm{~h}$ da manhã.

As regressões lineares entre os fluxos de seiva e as radiações de ondas curtas e fotossinteticamente ativas apresentaram bons coeficientes (Tabela 5). Os valores, como esperado, diminuíram nos cálculos realizados para a árvore B381 em janeiro de 2003, em relação ao período de dezembro de 2000, pois este exemplar passara mais de um ano sob restrição hídrica.

\section{CONCLUSÕES}

1. Este estudo revelou que as árvores de grande porte são suscetíveis a períodos de seca prolongada.

2. As medidas de fluxo de seiva no exemplar de Eschweilera Coriacea (B381) registraram reduções significativas na transpiração, após o período de seca.

3. A árvore A237 de Eschweilera Coriacea, que não foi submetida a limitação hídrica, registrou aumento na transpiração, entre os dois períodos estudados.

4. A diminuição da disponibilidade de água no solo prevaleceu como fator determinante da redução da transpiração na árvore B381, apesar da grande quantidade de energia disponível.

5. Um teor menor de água no solo disponível às plantas alterou o transporte de nutrientes para o vegetal e provocou reações, como a aceleração do processo de senescência e a queda atípica de folhas.

6. Notaram-se reduções no conteúdo de água no solo nas duas parcelas experimentais, entre os dois períodos analisados.

\section{AgRADECIMENTOS}

Agradecemos ao Grupo de Trabalho de Campo do MPEG em Breves e Caxiuanã, Pará, pela incondicional colaboração nas atividades realizadas na ECFPn. Aos revisores, pelas contribuições pertinentes. $\mathrm{O}$ primeiro autor agradece também à CAPES, pela concessão da bolsa de doutorado.

\section{LITERATURA CITADA}

Almeida, S.S.; Lisboa, P.L.B.; Silva, A.S.L. Diversidade florística de uma comunidade arbórea na Estação Científica Ferreira Penna, Caxiuanã, Pará. Boletim Museu Paraense Emílio Goeldi, Série Botânica, Belém, v.9, n.1, p.99-105, 1993.

Azevedo, P.V.; Silva, B.B.; Silva, V.P.R. Water requirements of irrigated mango orchards in northeast Brazil. Agricultural Water Management, Amsterdam, v.58, p.241-254, 2003.

Bonato, C.M.; Rubin Filho, C.J.; Melges, E.; Santos, V.D. Nutrição mineral de plantas. Maringá: UEM 1998, 60p.
Casa, R.; Russell, G.; Lo Cascio, B. Estimation of evapotranspiration from a field of linseed in central Italy. Agricultural and Forest Meteorology, Amsterdam, v.104, n.1, p.289-301, 2000.

Costa, R. F. da; Costa, A. C. L. da; Meir, P.; Alan P. B.; Malhi, Y. S.; Gonçalves, P. H. L.; Silva Jr., J. A.; Oliveira, P. J. Variação da precipitação pluviométrica no Sítio Experimental do LBA na Floresta Nacional de Caxiuanã, Pará, Brasil (20022003). In: International Scientific Conference of LBA, 3, 2004, Brasília.Resumos...Brasília: LBA, 2004, CD-Rom

Costa, R. F. da; Costa, A. C. L. da; Meir, P.; Malhi, Y.; Fisher, R. A.; Braga, A. P.; Oliveira, P. J.; Silva Junior, J. A.; Gonçalves, P. H. L.; Costa, J. M. N.; Vale, E. D. Sotta, J. C.; Cohen, R. L.; Ruivo, M. L.; Silva, R. B. C.; Andrade, V. M. S.; Grace, J. Projeto LBA/ESECAFLOR em Caxiuanã: Características, atividades e resultados. In: Seminário de 10 Anos de Atividades da ECFPn, Caxiuanã, 1, 2003, Belém. Resumos...Belém: MPEG, 2003a, CD-Rom

Costa, R. F. da; Meir, P.; Oliveira, P.J.; Malhi,Y.; Costa, A.C.L.; Costa, J.M.N; Silva, R.B.C.; Andrade,V.M.S.; Braga, A.; Gonçalves, P.H.L ; Silva-Júnior, J.A. Transporte de seiva em espécies vegetais de um ecossistema de manguezal Bragança, Pará, Brasil. In: Conferência Internacional Mangrove, 2, 2003, Salvador. Articulando a pesquisa com a gestão ambiental participativa de estuários e manguezais. Resumos... Recife: INTERGRAF, 2003b, v.1. p.175.

Èermák, J.; Kuèera, J.; Nadezhdina, N. Sap flow measurements with some thermodynamic methods, flow integration within trees and scaling up from sample trees to entire forest stands. Trees-Structure and Function, Berlim, v.18, p.529-546, 2004.

Fisch, G.; Marengo, J.; Nobre, C.A. Uma revisão geral do clima da Amazônia. Acta Amazônica, Manaus, v.28, n.2, p.101126, 1998.

Kern, D.C. Geoquímica e pedoquímica em sítios arqueológicos com terra preta na Floresta Nacional de Caxiuanã, Portel, PA. Belém: UFPA, 1996. 119p. Tese Doutorado

Köstner, B. Evaporation and transpiration from forests in Central Europe - relevance of patch-level studies for spatial scaling. Meteorology and Atmospheric Physics, Viena, v.76, p.69-82, 2001.

Kuèera, J. Sap Flow Meter - P4.1, Environmental measuring systems instruction manual. Brno: EMSBRNO, 1998. 20p.

Lisboa, P.L.B. Caxiuanã. Belém: Museu Paraense Emílio Goeldi. 1997. 446p.

Malhi, Y.; Meir, P.; Brown, S. Forests, carbon and global climate. Philosophical Transactions of the Royal Society, London. v.360, n,1797, p.1567-1591, 2002.

Marques, J.; Salati, E.; Santos, J.M. Cálculo da evapotranspiração real na Bacia Amazônica através do método aerológico. Acta Amazônica, Manaus. v.10, n.2, p.357-361, 1980.

Meinzer, F.C.; Andrade, J.L.; Goldstein, G.; Holbrook, N.M.; Cavelier, J.; Jackson, P. Control of transpiration from the upper canopy of a tropical forest: The role stomatal, boundary layer and hydraulic architecture components. Plant, Cell and Environment. Oxford, v.20, p.1242-1252, 1997. 
Meir, P.; Costa, A.C.L.; Costa, R. F. da.; Fisher, R. A.; Sotta, E. D.; Vale, R. L.; Silva Junior, J. A.; Almeida, S.S.; Ruivo, M.L.P.; Costa, J. M. N.; Carvalho, C.; Maroco, J.; Chaves, M.; Williams, M.; Malhi, Y.; Grace, J. Effects of experimental drought on carbon cycling in an Eastern Amazon rain forest. In: Science Team Business Meeting. 7, 2003, Fortaleza. Proceedings...Results of Ecological Studies of LBA. Fortaleza: LBA/ECO, 2003, CD-Rom.

Meir, P.; Costa, A.C.L.; Costa, R. F. da; Malhi, Y.; Fisher, R.A.; Costa, J.M.N.; Vale, R.L.; Sotta, E.D.; Ruivo, M.L.P.; Grace, J.Drought-sensitivity in the E. Amazon. In: International Scientific Conference of LBA, 2, 2002, Manaus. Proceedings...Manaus: LBA, 2002, CD-Rom.

Möller, M.; Tanny, J.; Li, Y.; Cohen, S. Measuring and predicting evapotranspiration in an insect-proof screenhouse. Agricultural and Forest Meteorology, Amsterdam, v.127, p.35-51, 2004.

Naves-Barbiero, C.C.; Franco, A.C.; Bucci, S.J.; Goldstein, G. Fluxo de seiva e condutância estomática de duas espécies Lenhosas sempre-verdes no campo sujo e cerradão. Revista Brasileira de Fisiologia Vegetal, Lavras, v.12, n.2, p.119134, 2000.

Netto, J.A.; Azevedo, P.V.; Silva, B.B.; Soares, J.M.; Teixeira, A.H.C. Exigências hídricas da videira na região do submédio São Francisco. Pesquisa Agropecuária Brasileira, Brasília, v.35, n.8, p.1559-1566, 2000.

Nicolasa, E.; Torrecillasa, A.; Ortuno, M.F.; Domingo, R.; Alarcón, J.J. Evaluation of transpiration in adult apricot trees from sap flow measurements. Agricultural Water Management, Amsterdam, v.72, p.131-145, 2005.

Orgaz, F.; Fernández, M.D.; Bonachela, S.; Gallardo, M.; Fereres, E. Evapotranspiration of horticultural crops in an unheated plastic greenhouse. Agricultural Water Management, Amsterdam, v.72, p.81-96, 2005.

Ruivo, M.L.P.; Pereira, S.B.; Bussetti, E.P.C.; Costa, R. F. da; Quanz, B.; Nagaishi, T. Y.; Oliveira, P. J.; Meir, P.; Malhi, Y.; Costa, A. C. L. Propriedades do solo e fluxos de $\mathrm{CO}_{2}$ em Caxiuanã, Pará: Experimento LBA-ESECAFLOR. In. Contribuiçoes a geologia da Amazonia, (CGA-3), Sociedade Brasileira de Geologia, Belém, v.3, p.291-299, 2002.
Sammis, T.W.; Mexal, J.G.; Miller, D. Evapotranspiration of flood-irrigated pecans. Agricultural Water Management, Amsterdam, v.69, p.179-190, 2004.

Shuttleworth, W.J.; Gash, J.H.C.; Lloyd, C.R. ; Roberts, J. M.; Molion, L.C.B.; Nobre, C.A.; SÁ, L.D. de A.; Marques Filho, A de O.; Fisch, G.; Januário, M.; Fattori, A.P.; Ribeiro, M.N.G.; Cabral, O.M.R. Amazonian evaporation. Revista Brasileira de Meteorologia, São Paulo, v.2, n.1, p.179-191, 1987.

Silva, A.S.L.; Almeida, S.S.; Rosário, C.S. Flórula fanerogâmica da Estação Científica Ferreira Penna (ECFPn): Caracterização dos ecossistemas e lista preliminar de espécies. In: Seminário de 10 anos de atividades da ECFPn, Caxiuanã, 1, 2003, Belém. Resumos... Belém: MPEG, 2003, CD-Rom.

Silva, B.B; Slack, D.C.; Kilby, M.W.; Hla, A.K. Measurements of water requirements of table grapes in Arizona (USA). Revista Brasileira de Agrometeorologia, Santa Maria, v.8, n.2, p.175-183, 2000.

Testi, L.; Villalobos, F.J.; Orgaz, F. Evapotranspiration of a young irrigated olive orchard in southern Spain. Agricultural and Forest Meteorology, Amsterdam, v.121, p.1-18, 2004.

Viana, J.S.; Almeida, S.S.; Conceição, C.; Ferreira, E.; Alves, N.; Silva,R. Comparação estrutural e florística entre os ambientes de Terra-Firme e Igapó do entorno da Estação Científica Ferreira Penna - ECFPn. In: Seminário de 10 anos de atividades da ECFPn, Caxiuanã, 1, 2003, Belém. Resumos... Belém: MPEG, CD-ROM, 2003.

Villa Nova, N.A.; Salati, E.; Matsui, E. Estimativa da evapotranspiração na Bacia Amazônica. Acta Amazônica, Manaus, v.6, n.2, p.215-228, 1976.

Williams, D.G.; Cable, W.; Hultine, K.; Hoedjes, J.C.B.; Yepez, E.A.; Simonneaux, V.; Er-Raki, S.; Boulet, G.; de Bruin, H.A.R.; Chehbouni, A.; Hartogensis, O.K.; Timouk, F. Evapotranspiration components determined by stable isotope, sapflow and eddy covariance techniques. Agricultural and Forest Meteorology, Amsterdam, v.125, p.241-258, 2004. 